stösst allerdings auf Schwierigkeiten, da die Merkmale im Blutserum wenig konstant sind. Im wesentlichen sind die Schwankungen durch das Grundfutter bedingt, wodurch z.T. Jahres. saisonale und betriebliche Unterschiede hervorgerufen werden. Die daraus entstehende Problematik wird am Beispiel mehrjähriger Untersuchungen erläutert.

\title{
ERBLICHKEITSSCHÄTZUNG FÜR VERSCHIEDENE METABOLITEN IM BLUTSERUM VON BULIEN
}

\section{N. H. FiSCHER. - Institut f. Tierhygiene Bonn, BRD.}

An I092 Bullen wurden folgende Serumbestandteile gemessen : die Aktivität der GlutamatOxalacetat Transaminase (GOT), das Gesamtcholesterin, die wahre Glucose, anorganisches Phosphat, Calcium, Natrium und Kalium. Bei allen Mineralstoffen zeigten sich hochsignifikante jahreszeitliche Unterschiede. Bei der GOT-Aktivität, dem Gesamtcholesterin und der GIucose war dieser Effekt nicht so ausgeprägt und regional unterschiedlich. Nur bei der GOT-Aktivität und der Glucose zeigten sich auch Unterschiede zwischen den Zuchtverbänden. Die Kovariablen Alter, Gewicht und durchschnittliche Milch-und Fettleistung der Mutter waren in ihrem Einfluss auf die Metaboliten sehr unterschiedlich.

Die geschätzten Heritabilitätskoeffizienten sind für : die GOT-Aktivität o, I 8 ; das Gesamtcholesterin 0,30 ; die Glucose 0,26 ; anorg. Phosphat o ; Calcium 0,33 ; Natrium o, I 2 ; und Kalium o. Die phänotypischen Korrelationen zwischen den Serumwerten waren unbedeutend.

Bej GOT zu Cholesterin, GOT zu Glucose und Cholesterin zu Calcium wurden genetische Korrelationen in der Grössenordnung von 0,4 geschätzt. Dabei war jedoch der Standardfehler sehr hoch, so dass keine eindeutige Beziehung angenommen werden kann.

\section{Systèmes planifiés de croisement chez le Porc}

\section{LES BASES DU CROISEMENT CHEZ I.E PORC}

\author{
P. Sellier. - Station de Génétique quantitative et appliquée, I. N. R. A., 78350 Jouy en Josas, \\ France.
}

Les différents paramètres du croisement sont passés en revue, dans,la ligne de l'analyse de Dickerson (1969). Le gain génétique résultant du croisement a une double origine : complémentarité, hétérosis. L'importance pratique de chacun de ces effets est évaluée, dans le cas du Porc. Des valeurs moyennes de l'hétérosis individuel et de l'hétérosis maternel pour les principaux caractères sont déduites d'estimations expérimentales présentées sous forme graphique. Il apparaît que, chez le Porc, les effets d'hétérosis, dont la part exploitée dans différents plans de croisement est rappelée, expliquent la majeure partie du gain lié au croisement. La variation observée des estimées d'hétérosis pour un caractère est analysée : variance d'échantillonnage, aptitude spécifique à la combinaison de certains couples de races, interactions de type hétérosis $\times$ milieu liaison entre hétérosis et moyenne parentale; quelques illustrations sont données. Enfin les différents critères utilisés en matière d'optimisation des stratégies de croisement chez le Porc sont présentés : la tendance actuelle est de ne plus rechercher seulement la productivité maximum de l'étage terminal du plan de croisement mais de s'intéresser au système de croisement dans son ensemble, soit à l'équilibre démographique, soit dans la phase de mise en place. Les premiers résultats d'une telle étude prévisionnelle concernant deux races françaises sont donnés.

\section{The EVAluation OF HYBRID PIGS}

D. M. Smith, D. E. Steane. - Meat and Livestock commission, Bletchley, Buckinghamshive, England.

British hybrid pig testing, known as Commercial Product Evaluation, aims to provide an independent assessment of stock offered for sale by breeding companies. In 1972-73 pigs were purchased from eight commercial companies, together with pure Large White and unimproved 